\title{
ION BEAM SHEPHERD SATELLITE FOR SPACE DEBRIS REMOVAL
}

\author{
M. Merino, E. Ahedo, C. Bombardelli, H. Urrutxua, \\ and J. Peláez \\ Universidad Politécnica de Madrid \\ Madrid 28040, Spain
}

\begin{abstract}
A novel contactless space debris removal system is proposed in which the debris orbital motion is actively controlled by exploiting the momentum transmitted by a collimated beam of quasi-neutral plasma impinging against its surface. The beam can be generated with state-of-the-art ion engines from a "shepherd spacecraft" coorbiting nearby. This spacecraft, placed at a distance of 10-20 m from the object, would use a state-of-the-art ion thruster pointed towards the debris, as well as a second propulsion system to compensate for the beam reaction in order to keep a constant distance between the debris and the shepherd satellite throughout the deorbiting process. The key aspects of the concept, including the propulsion requirements, the modeling of the plasma beam, the momentum transfer to the body, and the dynamics and control of the system, are discussed.
\end{abstract}

\section{INTRODUCTION}

The number of space debris in Low (LEO) and Geostationary (GEO) Earth orbits is increasing at an alarming pace. This poses a serious threat for the continued exploitation of space as a strategic resource for many scientific and commercial missions. Impacts in orbit occur at typical velocities of $10 \mathrm{~km} / \mathrm{s}$, meaning that a regular 1-centimeter object can pierce any vehicle unless strongly shielded. Although catastrophic collision probabilities are still currently low, a plausible chain reaction of collisions between debris could result in hundreds of thousands of fragments that would render such orbits unusable in practice for decades or centuries (scenario known as "Kessler Syndrome" [1]). Worryingly, current studies predict that the number of debris in the orbits of interest will continue to increase unavoidably during the next years - even if no further launch were made, due to collisions and explosions of existing pieces - unless active removal actions are undertaken. Active Debris Removal is, however, a difficult and risky

This is an Open Access article distributed under the terms of the Creative Commons Attribution License 2.0, which permits unrestricted use, distribution, and reproduction in any medium, provided the original work is properly cited. 
operation. Typically, the target space debris has to be docked or caught by another spacecraft before a deorbiting or reorbiting maneuver can take place. Docking with a noncooperative target, which, in addition, may exhibit chaotic attitude motion, is a formidable task in space technology.

Recently, our research team has proposed a novel contactless debris removal strategy, which can greatly reduce the complexity, costs, and risks of an active removal campaign by completely avoiding the technological challenge of physically docking to an uncooperative target [2-4]. A high-specific-impulse ion beam, produced onboard of a "shepherd" spacecraft, is used to exert a decelerating force on selected pieces of debris in order to effectively deorbit them from a safe distance. This shepherd, which directs the ion beam towards the target debris, is equipped with a secondary propulsion unit to counteract the reaction force of the primary beam, allowing to maintain a constant separation from the space debris throughout the deorbit (or reorbit) process. Similar concepts have been proposed independently by other research groups [5-7].

The same Ion Beam Shepherd (IBS) concept has also other potential propulsive applications of large interest which are currently under study. Preliminiary results suggest remarkable performances as an asteroid deflection system [8], with multiple advantages over other alternatives such as weighty gravity tractors [9] or difficult to control kinetic impactors [10]. Another envisaged use of the IBS is the controlled displacement of space assets in orbit which lack their own propulsion systems.

This paper describes the key aspects of the IBS concept and presents some recent results of an ongoing research effort on the system performances. The rest of the paper is structured as follows: section 2 summarizes the IBS system and discusses its basic performances. In section 3 , the specific needs of the IBS in terms of propulsion are analyzed. Section 4 explains the mathematical model used to study the plasma plume, the central element of the IBS-debris interaction. Then, the method followed to calculate the forces and torques exerted upon the target debris by the plasma is described in section 5. Lastly, the IBS concept and the capacity to control a deorbiting maneuver is illustrated with preliminary simulations in section 6 , and conclusions are gathered in section 7 .

\section{THE ION BEAM SHEPHERD CONCEPT}

The IBS $[2,3]$ is a new concept of space propulsion in which a shepherd spacecraft employs a primary propulsion system (e.g., an ion thruster) to produce and aim a collimated quasi-neutral plasma beam towards a target object. As a consequence, the latter receives a force $\boldsymbol{F}_{D}$, resulting from the momentum transmitted by the plasma ions when they reach its surface (Fig. 1). The force $\boldsymbol{F}_{D}$ is a fraction of the total thrust $\boldsymbol{F}_{p 1}$ produced by the primary propulsion system: 


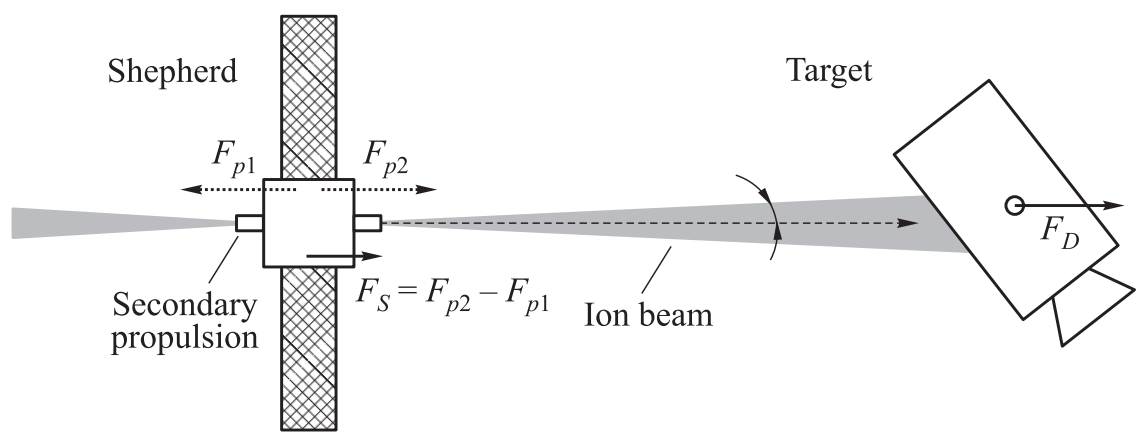

Figure 1 Schematic of IBS satellite deorbiting a space debris

$$
\boldsymbol{F}_{D}=-\eta_{B} \boldsymbol{F}_{p 1}
$$

where $\eta_{B}$ is the beam momentum transfer efficiency, one of the key figures of the system, which depends primarily on the problem geometry (relative distance, debris size, shape and attitude, and beam divergence rate), and, secondarily, on the characteristics of the debris surfaces.

In order to avoid that the IBS drifts away from the debris secularly, the thrust force $\boldsymbol{F}_{p 1}$ exerted on the shepherd needs to be compensated by a secondary propulsion system (e.g., another ion thruster), which delivers a force $F_{p 2}$ aimed in the opposite direction (see Fig. 1). In addition, the secondary propulsion system can be used to control the IBS-debris relative displacement along the orbit normal and along the out-of-plane direction.

Assuming that $F_{p 1}, F_{p 2}$, and $F_{D}$ are aligned with the tangent to the orbit, zero secular variation of the distance between the two bodies is obtained when their orbit semimajor axes are equal at any instant of time $t$, which translates into [3]:

$$
\frac{F_{D}}{m_{D}}=\frac{F_{S}}{m_{S}}
$$

where $F_{S}=F_{p 2}-F_{p 1}$ is the resulting force on the IBS, and $m_{D}$ and $m_{S}$ are the masses of debris and shepherd, respectively. Using Eq. (1), this condition reads:

$$
F_{p 2}=F_{p 1}\left(1+\eta_{B} \frac{m_{S}}{m_{D}}\right) .
$$

Note that the separation distance is ideally constant only for the case of a circular orbit; in the case of elliptic orbits, it oscillates about a mean value. The amplitude of the oscillations relative to this value is equal, to first order, to the orbit eccentricity. This makes the IBS concept less suitable for highly eccentric orbits. Fortunately, the great majority of large LEO debris has eccentricity less than 0.01 [3]. 


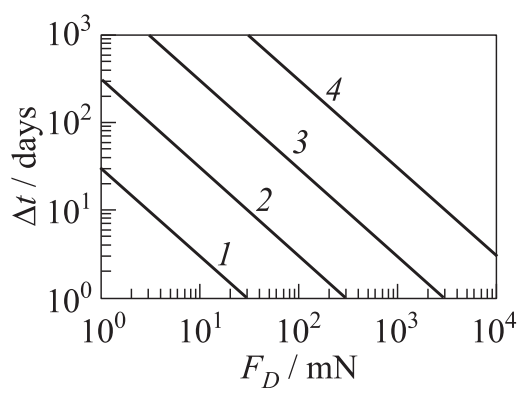

Figure 2 Deorbiting time for a low-thrust transfer from 1000- to 500-kilometer altitude circular orbit assuming constant tangential thrust $F_{t}$ and for different values of the space debris mass: $1-10 \mathrm{~kg} ; 2-100 \mathrm{~kg} ; 3-1 \mathrm{t}$; and $4-10 \mathrm{t}$

If the assumption is made that the debris orbit is initially circular and undergoes a constant tangential acceleration $F_{D} / m_{D}$, the time required to transfer the debris from a higher altitude orbit of radius $r_{1}$ to a lower altitude orbit of radius $r_{2}$ (or vice versa) is very accurately described by the following equation [2]:

$$
\Delta t=\Delta V \frac{m_{D}}{F_{D}}=\frac{m_{D}\left|\sqrt{r_{1}}-\sqrt{r_{2}}\right|}{F_{D} \sqrt{r_{1} r_{2} / \mu}}
$$

where $\mu$ is the Earth gravitational parameter, and $\Delta V$ is the mission velocity budget.

Figure 2 plots the time required for a 500-kilometer altitude reduction of debris of different sizes starting from a circular 1000-kilometer altitude orbit and as a function of the constant tangential thrust. A continuous 100-millinewton force seems to be reasonable in order to have 1-ton objects deorbited in about one month time. Assuming a specific impulse of $I_{\mathrm{sp}}=2700 \mathrm{~s}$ for both the primary and secondary thrusters and a beam momentum transfer efficiency $\eta_{B} \simeq 0.8$, such deorbiting maneuver would consume about $25-35 \mathrm{~kg}$ of propellant, both thrusters considered. Note that there are currently almost 1000 objects in LEO with a mass exceeding $1 \mathrm{t}$ and having reached the end of their operation time.

\section{PROPULSION REQUIREMENTS}

The whole IBS concept relies on the ability to transmit a sufficient deorbiting force to a distant body with an ion beam. It is, therefore, critical to analyze the main requirements for the propulsion system and discuss the different alternatives that can be used to generate the beam. To achieve this in a highly efficient manner, we require a plasma source able to generate $(i)$ a high specific impulse, $I_{\mathrm{sp}}$, with a high thrust efficiency: 


$$
\eta_{T}=\frac{I_{\mathrm{sp}} g_{0} F_{p 1}}{2 P_{a 1}}
$$

where $P_{a 1}$ is the available power for the primary ion beam source, and $g_{0}$ is the gravity acceleration at Earth surface, and (ii) a low divergence plasma jet.

While a larger value of $I_{\mathrm{sp}}$ means lower propellant consumption for a mission of given $\Delta V$, the available power on board, $P_{a}=P_{a 1}+P_{a 2}$, with $P_{a 2}$ $=\left(1+\eta_{B} m_{s} / m_{D}\right) P_{a 1}$, and the deorbiting design time, $\Delta t$, set a constraint to the maximum possible $I_{\mathrm{sp}}$. Assuming constant $F_{p 1}$ and $\eta_{B}$ throughout the mission and that both the primary and secondary propulsion systems have the same $I_{\mathrm{sp}}$ and thrust efficiency $\eta_{T}$, this constraint reads

$$
I_{\mathrm{sp}}=\frac{2 \eta_{T} \eta_{B} P_{a} \Delta t}{\Delta V\left(2 m_{D}+\eta_{B} m_{S}\right) g_{0}} .
$$

This expression manifests the importance of $P_{a}$ and $\eta_{T}$ for a swift and efficient deorbiting campaign.

The second main factor paramount for this application is the divergence angle of the beam. Given a working distance from the shepherd satellite to a certain target debris, the divergence angle controls the amount of plasma intercepted by the object, thereby determining the beam momentum transfer efficiency $\eta_{B}$. The smaller the divergence angle, the higher $\eta_{B}$ for a given separation distance, or, equivalently, the further away the IBS can operate for a fixed value of $\eta_{B}$.

Other propulsion requirements include the ability to tailor the thrust produced by the device to allow adjusting the distance between the two orbiting bodies.

Finally, the characteristics of existing plasma thrusters and their technological maturity have also to be taken into account. The two most trusted and successful types of advanced electric propulsion are ion engines and Hall effect thrusters which are nowadays fully-developed, space-tested, and commercially available in the power range 500-5000 W. Current thrusters of both types with 2-3 kW provide around 100-millinewton thrust. Hall thrusters have larger divergence angles $\left(\sim 40^{\circ}\right)$ and lower specific impulses (1500-2500 s, normally) than ion engines (typically $<20^{\circ}$ and $\sim 3000 \mathrm{~s}$, respectively). However, Hall thrusters are more compact and rely on simpler power processing units than ion engines $[11,12]$.

All these differences, remarkably the smaller divergence angle, suggest that ion engines are more appropriate for the IBS concept. Nevertheless, both types of engines, as well as other well-established devices such as arcjets, can be potentially employed in the IBS. Idem can be said of electric propulsion technologies currently being developed, such as helicon thrusters with tunable magnetic nozzles [13] or magnetoplasmadynamic thrusters [14], whose performances and specific characteristics might entail additional benefits for this application. 


\section{PLASMA PLUME MODELING}

An adequate modeling of the plasma plume expansion is crucial for the study of the IBS concept. For the purposes of this preliminary analysis, self-similar

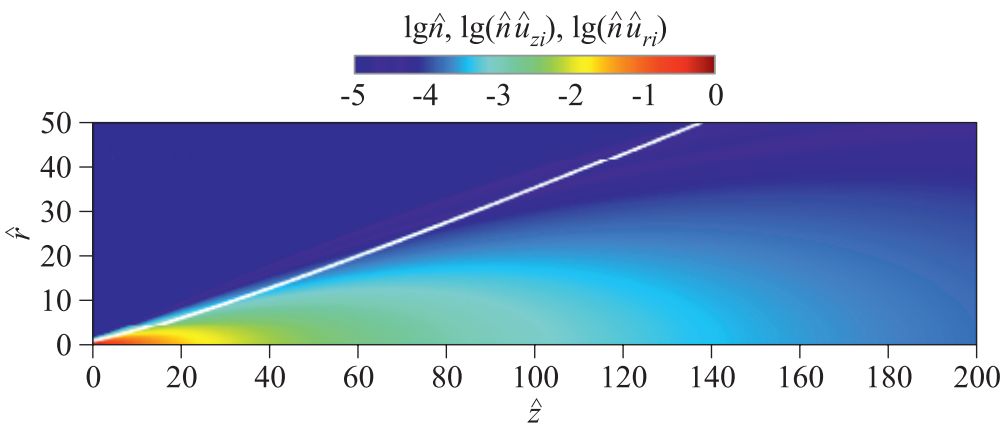

(a)

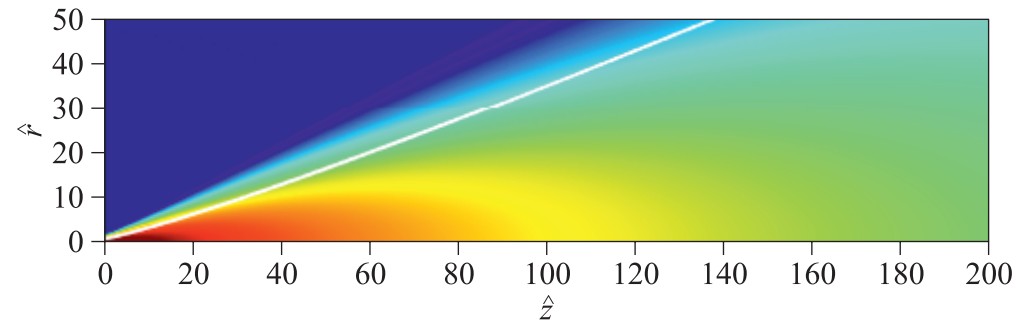

(b)

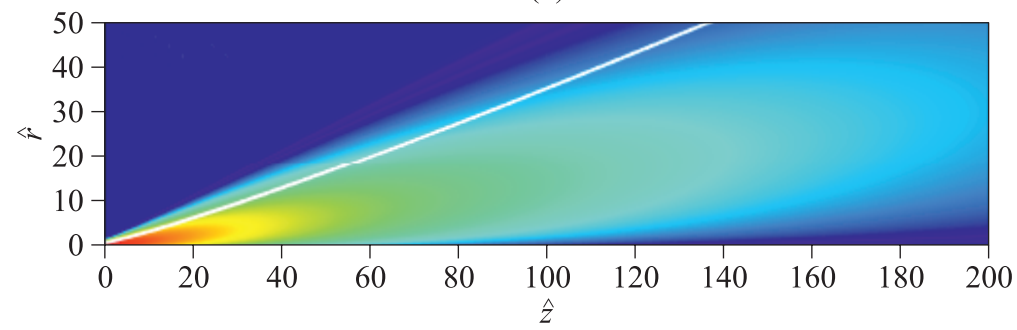

(c)

Figure 3 Profiles of the plasma density $\hat{n}(a)$, and the axial $\hat{n} \hat{u}_{z i}(b)$ and radial $\hat{n} \hat{u}_{r i}$ ion fluxes $(c)$ in the plasma plume in logarithm scale. The $\eta=1$ line (in white) represents the streamtube of 95 percent flux. The initial divergence angle of this line has been fixed at $\alpha_{0}=\tan ^{-1} 0.2$ and the Mach number at $\mathrm{M}_{0}=20$, representative value for ion thrusters [11, 12]. Variables with a hat (e.g., $\hat{u}_{z i}$ $=u_{z i} / \sqrt{T_{e} / m_{i}}$ ) are nondimensionalized with $T_{e}$ (electron temperature, expressed in energy units), $m_{i}$ (ion mass), $n_{0}$ (particle density at origin), and the initial radius $R_{0}$ of the plasma tube containing $95 \%$ of the mass flow. 
models (SSM) exhibit the best trade-off between accuracy and complexity, and retain all the relevant physics. These models provide a good estimation of the plasma plume properties in the far field [15], i. e., after the initial inhomogenities and thruster fields become negligible, which usually happens only a few thruster radii away from it [16]. For their simplicity and accuracy, SSM are under active investigation by our group. A detailed discussion and comparison of SSM can be found in [17] where the equations of these models are derived. A brief summary of the central aspects of SSM is provided below.

The SSM are based upon the steady-state continuity and radial momentum equations of the totally-ionized, collisionless plasma composed of singly-charged, hypersonic ions of mass $m_{i}$ and isothermal electrons of temperature $T_{e}$ :

$$
\begin{aligned}
u_{z i} \frac{\partial \ln n}{\partial z}+u_{r i} \frac{\partial \ln n}{\partial r}+\frac{\partial u_{z i}}{\partial z}+\frac{1}{r} \frac{\partial\left(r u_{r i}\right)}{\partial r}=0 ; \\
u_{z i} \frac{\partial u_{r i}}{\partial z}+u_{r i} \frac{\partial u_{r i}}{\partial r}+\frac{T_{e}}{m_{i}} \frac{\partial \ln n}{\partial r}=0
\end{aligned}
$$

where all the mentioned near-field effects have been neglected; $(r, \theta, z)$ are the set of cylindrical coordinates of the far field; $u_{z i}$ and $u_{r i}$ are the velocity axial and radial components; and $n$ is the plasma density.

Existing SSM neglect the axial plasma momentum equation and assume $u_{z i}$ $=$ const along streamlines. This makes the flow self-similar and induces a minor error, leaving the radial profile of the plasma indeterminate. Self-similar models differ in the additional assumptions on the shape of the plume upstream profiles. The plume expansion depends solely on the initial Mach number $\mathrm{M}_{0}=u_{z i 0}\left(m_{i} / T_{e}\right)^{1 / 2}$ and the initial divergence angle of the beam, $\alpha_{0}$.

Figure 3 displays the output of a particularization of the Ashkenazy and Fruchtman SSM [18] for a reference case, based on the currently-available ion thruster technology [11]: $R_{0}=0.1 \mathrm{~m}$ (the initial radius of the 95 percent massflow plasma tube) and $\mathrm{M}_{0}=20$ (representative of $u_{z i 0}=38000 \mathrm{~m} / \mathrm{s}$ and $T_{e}=5 \mathrm{eV}$ for Xenon). Notice that the expansion is not exactly conical due to the residual plasma pressure that continues to expand the beam downstream. The local relative error $\varepsilon$ (associated with ignoring the axial momentum equation and defined in [17]) committed by the model is less than $0.2 \%$ inside of the 95 percent mass flow tube. This error is proportional to $\mathrm{M}_{0}^{-2}$, making it negligible for hypersonic jets $\left(\mathrm{M}_{0} \gg 1\right)$ such as those of ion engines.

\section{FORCES AND TORQUES TRANSMITTED BY THE BEAM}

The high-speed plasma ions (typically, xenon) impact the target debris, exerting a force $\boldsymbol{F}_{D}$ upon the debris. This force has a main contribution associated to the 
momentum of the incoming ions per unit time. When ions reach the surface, a series of processes occur: according to experimental tests and numerical models available in the literature [19], the vast majority of ions with $O(1 \mathrm{keV})$ penetrate the substrate of metals such as aluminum a few nanometers and deposit their kinetic energy on the target via a series of collisions. The (neutralized) ions then abandon the surface after an accommodation time, with surface thermal velocities that are much smaller than the incoming ion velocity. Sputtering of the debris surface due to ion impact occurs, but the energy of the ejected materials is typically about two orders of magnitude smaller than the energy of the incoming ions. Although the particular accommodation and sputtering behavior depends on the material, its temperature, and its surface characteristics, as well as the energy of the impacting ions, these effects produce a negligible, positive contribution to the resulting force $\boldsymbol{F}_{D}$ in the ranges of application, and, therefore, are conservatively neglected. A preliminary analysis of these phenomena, as well as a discussion on the relative charging of the shepherd-debris system in the presence of the quasi-neutral plasma beam, can be found in [4]. The contribution of the static pressure of the plasma can also be neglected for hypersonic plumes.

Ultimately, the force on a differential surface element $d S$ of the target can be expressed to a good level accuracy as

$$
d \boldsymbol{F}_{D} \simeq m_{i} n \boldsymbol{u}_{i}\left(-\boldsymbol{\nu} \cdot \boldsymbol{u}_{i}\right) d S
$$

where $\boldsymbol{\nu}$ is the outwards-pointing normal unit vector of the surface element; $\boldsymbol{u}_{i}$ is the velocity vector of the incoming ions; and $n$ is their local density. The corresponding force $\boldsymbol{F}_{D}$ and torque $\boldsymbol{N}_{D}$ exerted by the plume can be, therefore, calculated by integrating over the debris surface $S_{b}$ exposed to the beam:

$$
\boldsymbol{F}_{D}=\int_{S_{b}} d \boldsymbol{F}_{D} ; \quad \boldsymbol{N}_{D}=\int_{S_{b}}\left(\boldsymbol{r}-\boldsymbol{r}_{G}\right) \times d \boldsymbol{F}_{D}
$$

where $\boldsymbol{r}-\boldsymbol{r}_{G}$ is the relative position of the integrating point with respect to the center of mass of the debris.

The plume model introduced in section 4 can be used to compute $\boldsymbol{F}_{D}$ and $\boldsymbol{N}_{D}$ for each geometric configuration. Since the backscattering accommodated ions and sputtered materials produce a negligible contribution and not all the ions reach the target surface, due to the beam divergence and possible misalignment, the beam momentum transfer efficiency $\eta_{B}$, defined in Eq. (1), is lower than unity.

As a simple illustration, a homogeneous spherical debris of radius $R_{D}$ and center-of-mass at $\left(r_{D}, z_{D}\right)$ is considered in the following. The transmitted force increases when the ratio between the sphere radius and the local radius of the beam $R_{B}$ (defined as the radius of the 95 percent mass flow streamtube at $z_{D}$ ) increases. The force also increases the smaller the misalignment $r_{D}$ of the center 
of mass from the axis of the beam. In the case $r_{D}=0$, the radial lateral force $F_{D, r}$ and the torque $\boldsymbol{N}_{D}$ are both zero. Figure 4 plots the beam efficiency vs. the impact ratio $\chi$,

$$
\chi=\frac{R_{D}}{R_{B}\left(z_{D}\right)}
$$

which is the function of the relative position of the two bodies, and for different values of the relative radial offset with respect to the beam width,

$$
\lambda_{r}=\frac{r_{D}}{R_{B}\left(z_{D}\right)} .
$$

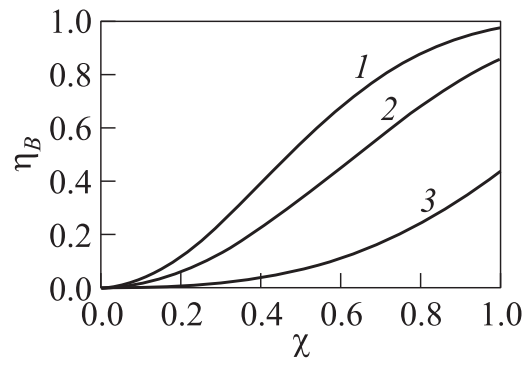

Figure 4 Beam momentum transfer efficiency for a sphere as a function of the impact ratio $\chi$ and the relative radial offset $\lambda_{r}=0(1), 0.5(2)$, and $1(3)$

These results highlight the importance of controlling the IBS-debris relative position in order to maximize $\chi$ and minimize $\lambda_{r}$ if a high value of $\eta_{B}$ is to be obtained. This translates into approaching closely the debris while maintaining a safe distance and optimizing beam divergence to reduce the fraction of plasma that does not impinge on the target.

\section{NUMERICAL SIMULATIONS AND CONTROLLABILITY}

The evolution of a deorbiting maneuver can be simulated using the elements introduced in previous sections to calculate the instantaneous force and torque on the two bodies. An in-house numerical simulation package called IBIS (Ion Beam Interaction Simulator), which can model the ion beam interaction with an arbitrary rigid object, was developed by our team. IBIS employs finite element methods together with a shadowing algorithm for the force and torque calculations, and was designed to deal with the specific dynamics arising from the IBS concept, combining the interaction of the plasma beam with the debris and the equations of motion for both the debris and the ion beam shepherd.

The IBIS software (Fig. 5) has become an important working tool for the tuning, testing, and validation of physical models, the study, analysis, and conceptual design of the IBS concept, as well as to evaluate and qualify the performance of the overall system, understand the influence of design parameters, and optimize particular features such as control laws and deorbiting strategies.

In the simulations shown here, the ion beam has been pointed along the shepherd orbit tangent. It produces a fixed thrust $F_{p 1}$ on the shepherd. To illustrate the IBS concept and its controllability, one will assume that the shepherd is 
equipped with three control thrusters (in the R-bar, V-bar, and out-of-plane direction) to control the relative position with the debris around a nominal value, corresponding to the debris and shepherd coorbiting at a user-defined separation distance. An optimally-tuned PD (proportional-derivative) control loop is
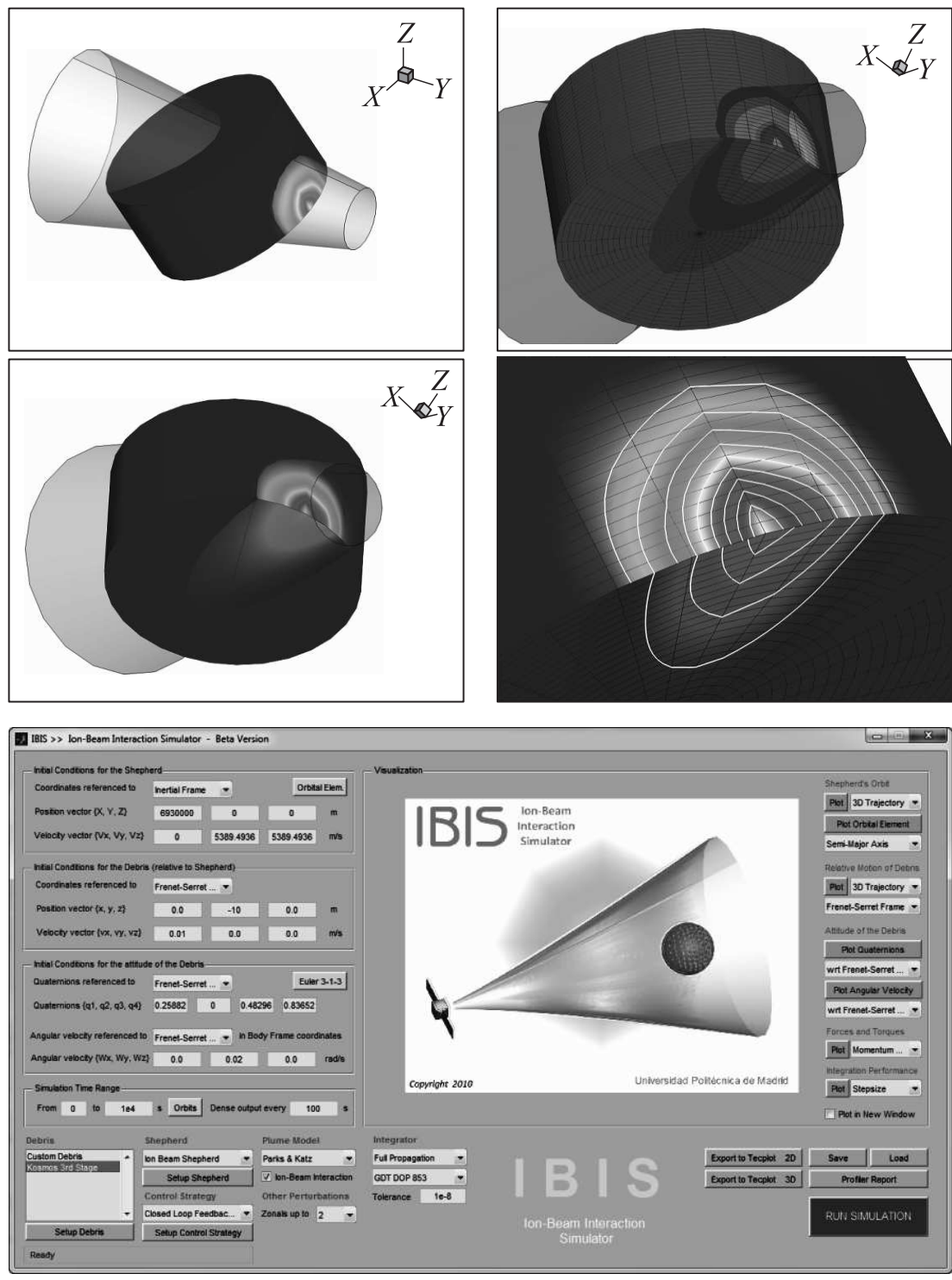

Figure 5 The IBIS software graphical output and graphic user interface 

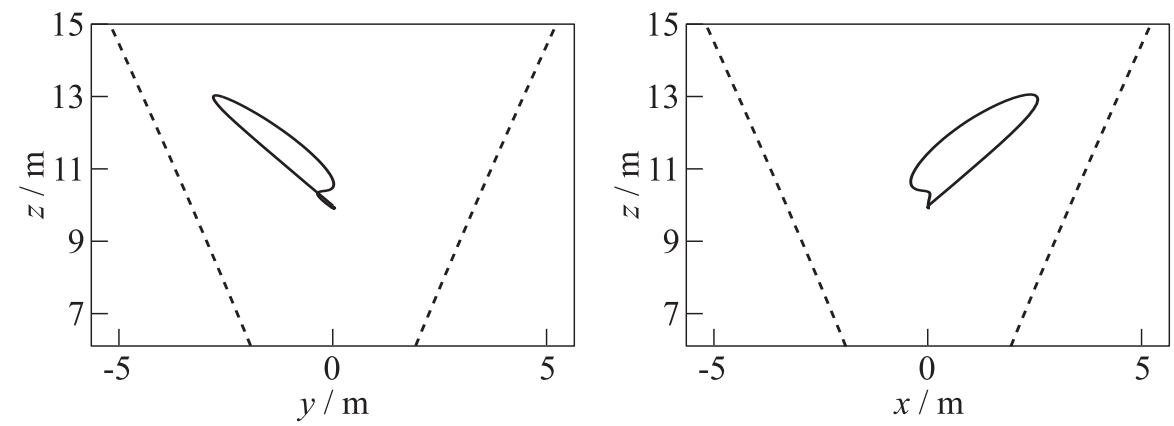

Figure 6 Relative trajectory of the center of mass of a 2-ton spherical debris in circular LEO orbit (altitude of $1000 \mathrm{~km}$ ) with $\chi=0.7$ subject to an initial impulse of $0.01 \mathrm{~m} / \mathrm{s}$ along the $x, y$, and $z$ axes from its nominal equilibrium position. The shepherd mass is set to $300 \mathrm{~kg}$. A beam of 0.1-newton (deorbiting) thrust and 10 degree divergence is employed. An optimally tuned PD feedback control system along the R-bar, V-bar, and out-of-plane direction was employed

employed to stabilize the relative motion. At this design phase, the availability of an exact measurement of the relative position between the two spacecraft has been assumed. Naturally, the chosen control strategy and the number/position of actuators are only preliminary and will be studied and optimized in detail together with the set of sensors used as the IBS concept advances.

Figures 6 to 8 show a representative simulation case with an initial relative velocity error from the nominal situation imposed in the initial conditions. The figures describe the initial evolution of the relative position of the two bodies, the magnitude of the control effort, and the debris angular velocity variation during the first orbits of the maneuver. As far as orbital perturbations are concerned, only J2 gravitational terms were accounted for. This simulation shows that the initial perturbation can be easily damped within the first few hours, after which the deorbiting maneuver can proceed successfully in a quasi-steady manner, in intervals of time as described by Eq. (2).

\section{CONCLUDING REMARKS}

A novel ion beam shepherd concept for active space debris removal operations, which can also work as a general contactless space-tug system and an asteroid deflection platform, has been succintly described. The system constitutes a promising and simple alternative to complex docking maneuvers. The evolution of a plasma plume from an electric propulsion system and the interaction between the plasma and the target debris have been studied. Preliminary results on the relative motion between the two bodies along a deorbiting maneuver using a 


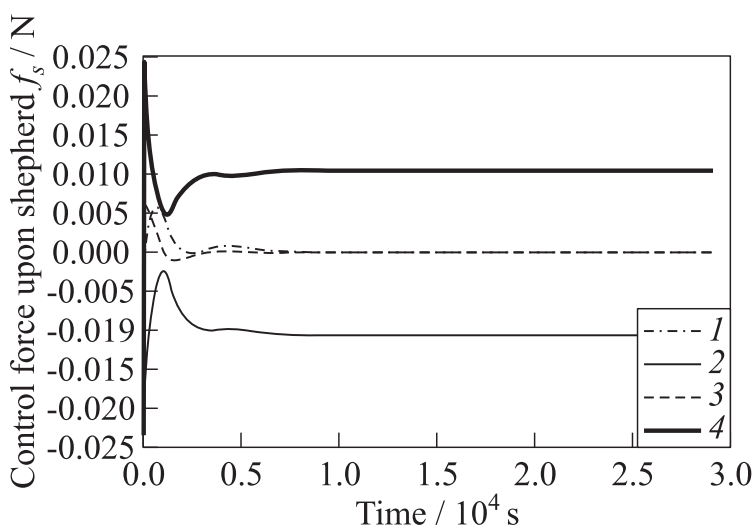

(a)

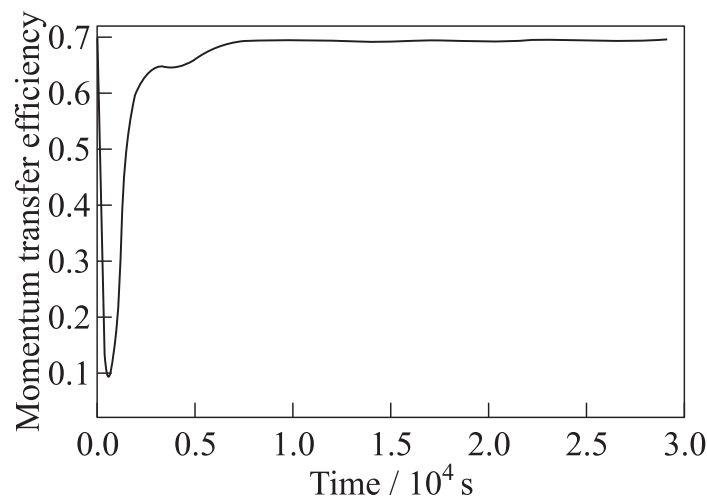

(b)

Figure 7 Control force components $(a)$ and momentum transfer efficiency $(b)$ for the relative position control problem described in Fig. 6: 1 - control force $f_{s}$ along $x$ axis in Frenet frame; 2 - along $y$ axis; 3 - along $z$ axis; and 4 - total control force

simple control strategy have been presented. The analysis, which relies on an ad-hoc developed ion beam interaction simulation package, shows that an ion beam can be used to exert forces and torques in a controlled manner from a safe distance on space debris, with momentum transfer efficiencies close to unity, and that formation flying of the IBS-debris system can be easily stabilized in quasi-circular orbits.

A detailed study of the system must follow, in order to gain a better understanding of $(i)$ the different phenomena in the plasma-body interaction; $(i i)$ the influence of the space environmental plasma in the momentum transmission; (iii) the effects of background magnetic fields such as the geomagnetic field on 


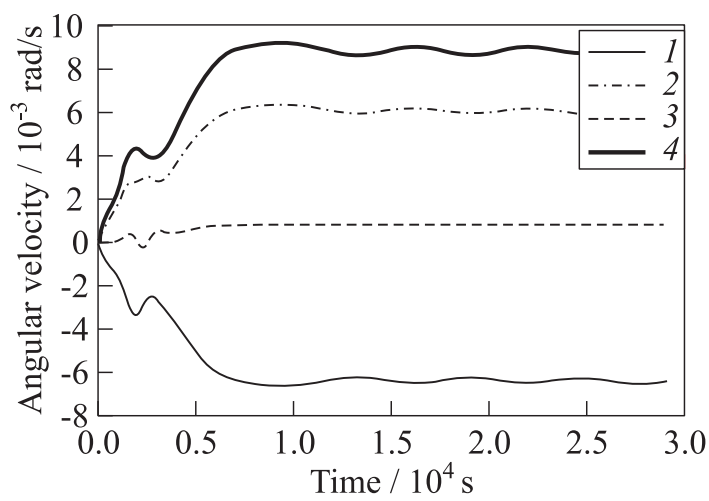

Figure 8 Evolution of the debris angular velocity vector (with respect to Frenet axes) for the relative position control problem described in Fig. 6: $1-w_{x} ; 2-w_{y} ; 3-$ $w_{z} ;$ and $4-|w|$

the plume expansion; and $(i \nu)$ assessing possible contamination effects on the IBS satellite due to backscattering particles.

In the area of dynamics and control, the issue of estimating the debris relative position and attitude with sufficient accuracy, as required by the control system, remains to be investigated. Future work will also assess the case of space debris of more complex shapes, and the possibility of stabilizing the attitude of the debris using the plasma beam, with a view to an eventual demonstration mission for the concept.

\section{ACKNOWLEDGMENTS}

This work was supported by the ARIADNA programme "Call for Ideas on Active Debris Removal," established by the Advanced Concepts Team of the European Space Agency (ACT-ESA), under contract No. 4000101447/10/NL/CBi. The final report of the project can be found in [20].

\section{REFERENCES}

1. Kessler, D. J., and B. G. Cour-Palais. 1978. Collision frequency of artificial satellites: The creation of a debris belt. J. Geophys. Res. 83(A6):2637-46.

2. Bombardelli, C., and J. Pelaez. 2011. Ion beam shepherd for contactless space debris removal. J. Guidance Control Dyn. 34(3):916-20.

3. Bombardelli, C., H. Urrutxua, M. Merino, E. Ahedo, J. Pelaez, and J. Olympio. 2011. Dynamics of ion-beam-propelled space debris. 22nd Symposium (International) on Space Flight Dynamics. Available online at web.fmetsia.upm.es/ep2. 
4. Merino, M., E. Ahedo, C. Bombardelli, H. Urrutxua, J. Pelaez, and L. Summerer. 2011. Space debris removal with an ion beam shepherd satellite: Target-plasma interaction. AIAA Paper No. 2011-6142.

5. Bonnal, C., J. M. Ruault, P. Bultel, and M. C. Desjean. 2010. High level requirements for an operational space debris deorbiter. 61st Astronautical Congress (International). IAC-10.A6.4.5.

6. Kitamura, S. 2010. Large space debris reorbiter using ion beam irradiation. 61st Astronautical Congress (International). IAC-10.A6.4.8.

7. Kitamura S., Y. Hayakawa, and S. Kawamoto. 2011. A reorbiter for GEO large space debris using ion beam irradiation. 32nd Electric Propulsion Conference (International). IEPC-2011-087.

8. Bombardelli, C., and J. Peláez. 2011. Ion beam shepherd for asteroid deflection. J. Guidance Control Dyn. 34(4):1270-72.

9. Wie, B. 2008. Dynamics and control of gravity tractor spacecraft for asteroid deflection. J. Guidance Control Dyn. 31(5):1413-23.

10. Ahrens, T. J., and A.W. Harris. 1992. Deflection and fragmentation of near-Earth asteroids. Nature 360:6403.

11. Martínez-Sánchez, M., and J.E. Pollard. 1998. Spacecraft electric propulsion — an overview. J. Propul. Power 14:5:688-99.

12. Ahedo, E. 2011. Plasmas for space propulsion. Plasma Phys. Controlled Fusion 53(12):124037.

13. Ahedo, E., and M. Merino. 2010. Two-dimensional supersonic plasma acceleration in a magnetic nozzle. Phys. Plasmas 17:073501.

14. Heimerdinger, D. J., and M. Martínez-Sánchez. 1988. Fluid mechanics in a magnetoplasmadynamic thruster. 20th Electric Propulsion Conference (International). IEPC-88-033.

15. Korsun, A. G., F.F. Gabdullin, E. M. Tverdokhlebova, and B.S. Borisov. 1999 Comparison between plasma plume theoretical models and experimental data. 26th Electric Propulsion Conference (International). IEPC-99-221.

16. Nakles, M. R., L. Brieda, D. R. Garrett, W. A. J. Hargus, and R. L. Spicer. 2007. Experimental and numerical examination of the BHT-200 hall thruster plume. AIAA Paper No. 2007-5305.

17. Merino, M., E. Ahedo, C. Bombardelli, H. Urrutxua, and J. Peláez. 2011. Hypersonic plasma plume expansion in space. 32nd Electric Propulsion Conference (International). IEPC-2011-086.

18. Ashkenazy, J., and A. Fruchtman. 2001. Plasma plume far field analysis. 27th Electric Propulsion Conference (International). IEPC-01-260.

19. Ziegler, J.F., J. P. Biersack, and M.D. Ziegler. 2008. SRIM - the stopping and range of ions in matter. Lulu Press. Ch. 7. 28.

20. Bombardelli, C., M. Merino, E. Ahedo Galilea, J. Peláez, H. Urrutxua, J. Herrera, A. Iturri, J. Olympio, L. Summerer, and D. Petkow. 2011. Active removal of space debris: Ion beam shepherd for contact-less debris removal. ARIADNA Project Final Report (10-6411c). ESA-ESTEC Advanced Concepts Team. Available online at web.fmetsia.upm.es/ep2. 